\title{
Images and Imagination: Automated Analysis of Priming Effects Related to Autism Spectrum Disorder and Developmental Language Disorder
}

\author{
Michaela Regneri ${ }^{1}$ \\ OTTO (GmbH \& Co. KG) \\ Hamburg, Germany \\ regneri@googlemail.com \\ Fahreen Walji ${ }^{1}$ \\ University of Roehampton \\ London, United Kingdom
}

\begin{abstract}
Different aspects of language processing have been shown to be sensitive to priming but the findings of studies examining priming effects in adolescents with Autism Spectrum Disorder (ASD) and Developmental Language Disorder (DLD) have been inconclusive. We present a study analysing visual and implicit semantic priming in adolescents with ASD and DLD. Based on a dataset of fictional and script-like narratives, we evaluate how often and how extensively, content of two different priming sources is used by the participants. The first priming source was visual, consisting of images shown to the participants to assist them with their storytelling. The second priming source originated from commonsense knowledge, using crowdsourced data containing prototypical script elements. Our results show that individuals with ASD are less sensitive to both types of priming, but show typical usage of primed cues when they use them at all. In contrast, children with DLD show mostly average priming sensitivity, but exhibit an overproportional use of the priming cues.
\end{abstract}

\section{Introduction}

This study compares the effects of priming on the narratives of adolescents with Autism Spectrum Disorder with those of adolescents with Developmental Language Disorders ${ }^{2}$. Priming occurs when exposure to one stimulus influences the response to a subsequent stimulus. This is an unconscious process whereby an association is activated in memory just before a new stimulus is seen or a task is introduced and is thought to play an important role in facilitating language fluency (MacDonald,

\footnotetext{
${ }^{1}$ The first and third authors conducted this research independently, this paper is not related to their current affiliations.

${ }^{2}$ Following recent recommendations (Bishop et al., 2016, 2017), the term Developmental Language Disorders has been adopted and used throughout in this paper.
}

\author{
Diane King \\ The Open University \\ Milton Keynes, United Kingdom \\ d.king@open.ac.uk \\ Olympia Palikara \\ University of Warwick \\ Coventry, United Kingdom \\ Olympia.Palikara@warwick.ac.uk
}

2013), implicit language learning (Dell and Chang, 2014), and in conversation and social interaction (e.g. Bresnan et al., 2007). Different aspects of language processing have been shown to be sensitive to priming (KIDD, 2012; Rämä et al., 2013) but whilst studies have typically examined priming effects in children (Foltz et al., 2015; Goldwater et al., 2011), there is a paucity of literature comparing priming effects in adolescents with ASD with those of adolescents with DLD.

Both ASD and DLD are communication disorders, with individuals in these clinical groups often having overlapping language phenotypes (Tomblin, 2011). However, it is unclear whether or not the language difficulties they exhibit have the same or different underlying aetiology (Manolitsi and Botting, 2011). ASD is a neurodevelopmental disorder characterised by impairments in social interaction, verbal and nonverbal communication and restricted and repetitive behaviours (APA, 2013). DLDs affect about $7.5 \%$ of children during their first year of school (Norbury and Bishop, 2003; Tomblin, 2011), and are associated with different language difficulties (e.g phonology, semantics and syntax). These impairments can be receptive, expressive or mixed (APA, 2013) and are likely to persist into adolescence and young adulthood (Conti-Ramsden and Botting, 1999; Dockrell et al., 2007).

Research has shown that both individuals with ASD and those with DLD produce narratives that are impoverished in comparison to their typically developing peers, displaying difficulties in both macrostructure and microstructure (Wetherell et al., 2007; Rezzonico et al., 2015; King et al., 2013, 2014). Studies comparing narratives of children from these two clinical groups have found few differences in structural language, evaluation and pragmatic measures (Norbury and Bishop, 2003; Manolitsi and Botting, 2011; Frazier Norbury et al., 2013). As yet, it is not clear if this is because both 
groups have the same underlying language difficulties, or if there are diagnostic specific factors affecting their narrative production.

Narrative difficulties of individuals with ASD may be explained, in part, by the information processing theory of weak central coherence. This theory proposes that something within the typical cognitive system derives meaning by relating incoming information to global context (Frith, 1989; Happe and Frith, 2006). Frith argued that individuals with ASD display differences to neurotypicals in this aspect of information processing, resulting in a tendency to focus on details at the expense of the whole. It is possible that the ability to make use of context to infer meaning is related to priming effects in language. If this is so, then we would expect individuals with ASD to display differences in priming compared to both their typically developing peers and the DLD group which would be reflected in their narrative language.

There is clearly a need for further research in this area and, to date, we can find none that compares priming effects in individuals with an ASD or DLD. We believe this to be an important area to explore for a number of reasons. A better understanding of the specific areas of difficulty experienced by both these clinical groups in not only structural, but also other forms of priming would inform the planning of appropriate intervention and support. Furthermore, if differences in priming are found between the two groups, this may give some indication as to whether or not the language difficulties they exhibit arise from the same or different underlying aetiology. Moreover, if individuals with ASD, but not those with DLD, display difficulties in priming, this may offer support for the theory of weak central coherence as an explanation for autism. In this study, our aim was to investigate priming effects in language production at the discourse level in adolescents with these communication disorders. In order to do so, we analysed visual and script based priming cues, and the extent to which these types of context present difficulties for individuals with ASD or DLD. A further aim was to examine whether these effects are related to language competency and development, or originated from typical traits of individuals with ASD.

This paper is structured as follows: After a review of related work (Sec. 2), we describe the dataset (Sec. 3) underlying our experiments (Sec. 4). After a discussion of the results (Sec. 5) we conclude with a short summary and suggestions for future research (Sec. 6).

\section{Related Work}

To date, research into priming effects on the language of individuals with either ASD or DLD, has primarily focused on structural or syntactic priming. The findings are inconclusive, but suggest that both these groups exhibit less ability to benefit from priming cues than typically developing children (Kamio et al., 2007). Mottron et al. (2001) have shown that individuals with ASD benefit more from phonological cues than semantic cues. Evidence from fMRI scans also suggests that the performance of individuals may depend on perceptual rather than semantic processing (Gaffrey et al., 2007). Hala et al. (2007) showed that for children with ASD, priming cues are rarely sufficient to change previously triggered meanings of homographs.

Studies of priming in individuals with DLD are similarly inconclusive. Miller and Deevy (2006) found that children with DLD displayed similar syntactic priming effects as typically developing children. However, another study using a structural priming paradigm (Garraffa et al., 2015) suggests that children with DLD show a smaller cumulative priming effect. Garraffa et al. (2018) later concluded that children with DLD exhibit patterns of syntactic priming effects which are consistent with an impairment in implicit learning mechanisms. In this experiment, none of the groups showed a significant cumulative priming effect.

Our investigation of priming effects required suitable raw data together with either extensive manual annotation or adequate computational methods. We decided to apply methods from the field of computational stylometry, which is concerned with the analysis of (written or transcribed) text and how it reveals information about the person who has produced this (Daelemans, 2013).

For DLD, there are virtually no approaches to automated analyses: Gabani et al. $(2009,2011)$ detected language impairment in Spanish texts, with a focus on the classification task itself rather than on stylometric analyses of explaining features.

There are, however, some studies where language difficulties typical in ASD have been subject to automated analysis. Most approaches have tried to automatically classify texts by individuals with ASD: Prud'hommeaux et al. (2011) built an auto- 
mated classifier for sentences uttered by very young children (6-7 years old), distinguishing between an ASD group and two comparison groups (one with children with a language impairment, one with typically developing children). The authors themselves note some drawbacks of their underlying dataset, in particular that some children in the ASD group were also classified as language-impaired. Consequently, it was not possible to demonstrate a clear distinction between the groups.

In two follow-up studies (Rouhizadeh et al. $2013,2015)$, the authors analysed whole narratives (retellings) of children (mean age 6.4) with ASD compared to a typically developing comparison group. The texts from the comparison group and some crowdsourced retellings from typically developing adults served as a basis for determining unusualness. We adopted parts of this idea and, in our study, collected data via crowdsourcing and matched it to the narratives under research. In our case, the collected data does not consist of whole narratives, but rather abstract prototypical facts.

Beyond the classification approaches, De Bruyne et al. (2018) present a detailed feature analysis of Dutch texts by adolescents with ASD, identifying shallow and deep features that indicate ASDrelated difficulties. Our own work (Regneri and King, 2015, 2016) also reports different shallow and discourse-based analyses of stories by children with ASD, compared to two control groups. We have used the same data source and have taken these analyses a step further. Moving beyond textbased analyses, we have targeted contextual priming effects. In addition to examining language typical for ASD, we have extended the dataset and also evaluated texts by adolescents with DLD.

\section{Datasets}

Our analysis was based on datasets by King et al. (2013, 2014) and by King and Palikara (2018a). The corpus contains transcripts of two types of stories: fictional stories and stories we call event narratives which describe common scenarios. Following is a description of the participant groups, the two story types and a short summary of the whole corpus.

\subsection{Participants and Experimental Setup}

The first cohort of participants comprised three groups: 27 high functioning adolescents with ASD (11 to 14 years old), one comparison group of
27 adolescents matched with the ASD group on chronological age and nonverbal ability ("agematched" peers, AM), and a second comparison group of 27 adolescents ( 7 to 14 years old), who were individually matched with the ASD group on a measure of expressive language ( $\mathrm{Re}$ calling Sentences subtest of the CELF IV (Semel et al., 2006)) and on nonverbal ability ("languagematched" peers, LM). All groups had average scores on non-verbal and verbal measures, as measured by the Matrices test of the BAS II (Elliot et al., 1996) and the BPVS II (Dunn and Dunn, 1997). There were no significant differences between the groups in measures of non-verbal ability, verbal ability or expressive language. The average age difference between the language-matched comparison group and the two other groups was 17 months. This dataset was extended by King and Palikara (2018b) with data from 25 adolescents with DLD, who were matched to the ASD group by age and non-verbal but not verbal ability. Children with Developmental Language Disorder often experience vocabulary difficulties and delays when compared to their typically developing peers (Gray et al., 1999; Rice et al., 2010; Rice and Hoffman, 2015). The profile of the children with DLD in this study on the BPVS is very much in line with that reported by other studies in the field, highlighting the deficits these children experience in receptive vocabulary when compared to other groups of children. See Table 1 for some basic characteristics of the three groups.

For data collection, each participant generated two fictional narratives and 12 different event narratives. The participants constructed these narratives following different prompts, accompanied with a supporting picture. In the experimental setup, fictional and event narratives were elicited in turn, with 6 event narratives followed by a fictional narrative. In our experiments, we used both the accompanying images and the script-like event scenarios as priming sources. The Appendix shows the instructions given to the participants, examples from the story collection and the image stimuli.

\subsection{Fictional Narratives}

For the fictional dataset, King et al. (2014) presented participants in all four groups with the following two story stems, along with a drawing, and asked them to continue the narrative:

1. The "forest" story: The boy ran into the forest. 


\begin{tabular}{lcccc}
\hline Group & ASD & DLD & LM & AM \\
Participants & 27 & 26 & 27 & 27 \\
\hline Age in years & 12.77 & 12.44 & 11.33 & 12.77 \\
SD & $(0.96)$ & $(1.56)$ & $(2.96)$ & $(0.82)$ \\
\hline BPVS II score & 104.50 & 75.19 & 110.93 & 107.14 \\
SD & $(20.97)$ & $(12.77)$ & $(13.22)$ & $(15.36)$ \\
\hline
\end{tabular}

Table 1: Mean age and test scores of ASD, DLD, Language Match (LM) and Age Match Group (AM). Standard deviation (SD) is shown in brackets.

\begin{tabular}{lcccccc}
\hline & \multicolumn{3}{c}{ words / text } & \multicolumn{3}{c}{ words / utterance } \\
& GEN & SEN & FIC & GEN & SEN & FIC \\
\hline ASD & 191 & 244 & 162 & 6.6 & 7.2 & 14.4 \\
DLD & 209 & 218 & 182 & 5.9 & 7.0 & 14.1 \\
LM & 252 & 400 & 220 & 10.5 & 10.2 & 16.9 \\
AM & 280 & 420 & 182 & 11.8 & 11.0 & 17.2 \\
\hline
\end{tabular}

Table 2: Text and utterance length of all narratives, by comparison group and narrative types.

\section{He looked ahead of him and saw a little green man in a spaceship.}

2. The "mountain" story: When the girl climbed up the mountain, she saw, hidden among the trees, a little wooden house covered in snow.

King et al. (2014) recorded the stories, transcribed, manually coded and scored them according to the Narrative Scoring Scheme (Stein and Albro, 1997).

\subsection{Event Narratives}

The corpus of event narratives (King et al., 2013) contains transcripts of short stories, describing one of 6 everyday scenarios each: SPENDING FREE TIME, BEING ANGRY WITH SOMEBODY, GOING ON HOLIDAYS, HAVING A BIRTHDAY, HALLOWEEN and BEING SCARED.

Every participant told two narratives for each of the scenarios: first a specific narrative (SEN) about a particular instance (answering a prompt like "Can you tell me about a time when you went on holiday?"), and second a general narrative (GEN) that contains a script-like prototypical scenario description ("What usually happens when someone goes on holiday?"). Overall, the set contains 1272 narratives $(2 \times 6$ stories per participant).

Table 2 presents basic statistics for the whole corpus. The figures distinguish the participant groups and additionally the fictional (FIC) narratives from the general (GEN) and specific (SEN) event narratives. The narratives from the ASD and the DLD group were, in general, shorter than those of the comparison groups, both with respect to the overall story length and the mean length of utterance.

\subsection{Contextual and Visual Priming}

The dataset allowed us to differentiate between issues of language development and ASD-specific difficulties, because it has two control groups matched accordingly. Content-wise, the stories enable research on specific priming effects in ASD and DLD, namely image-based priming, cued by visual situational context, and script-based priming, reflecting the influence of prototypical structures. Our study evaluated whether and how often both types of content appear in the narratives.

As a source for image-based priming, the corpus contains pictures used to support the adolescents' story telling. Our second source for priming was scripts: Scripts (Schank and Abelson, 1977) are pieces of commonsense knowledge describing everyday scenarios (e.g. going to a restaurant). They contain events (ordering, eating,...) and their participants (food, waiter, ...). Script-based priming means that a prototypical event representation is evoked and influences the story contents. To investigate related priming effects, we studied how much prototypical content is reflected in the adolescents' (specific) event narratives, and then related this to their overall ability to describe the prototypical scenarios (in the general event narratives).

\section{Priming Experiments}

We examined scripts and images as two different priming sources, and analysed the influence of these different contextual cues in narrations by adolescents with ASD, DLD and neurotypical developing peers. We thus distinguished the priming mechanisms that reflect the developmental stage of language competency (by comparing the languagematched group with the ASD group), fundamental differences in language development (comparing the DLD group with the other groups) or typical traits of individuals with ASD.

We will first present our definition of priming and the goals of our experiments (4.1). After describing how we crowdsourced textual representations of the different priming stimuli (4.2) and which measures we used to relate them to the narratives (4.3), we outline the results (4.4) 


\subsection{Priming from Images and Scripts}

Our experiments evaluated different aspects of priming. We used priming in a slightly unusual sense here, referring to the reflection of cues in the generation of narratives. The cues we examined were both nonlinguistic cues, originating from two different types of context.

\subsubsection{Visual priming from images}

We defined visual priming as the extent to which an image-based cue is reflected in a narrative that does not describe the image itself. In this study, participants were presented with pictures to support the construction of their narratives, but these might or might not be used for this purpose. Our focus was to evaluate to what degree this visual context affected the narratives produced by each group. We hypothesised that if the narratives of a specific group contained a large proportion of references to the image, this would indicate a heavy reliance on the picture as support. This suggests that they may be experiencing difficulties in constructing narratives independently and may indicate the presence of a developmental language disorder. On the other hand, if very little or no visual context is verbalised, we can infer that participants are having difficulties either processing or applying information from the images. It is of course also possible, that they simply choose not to use this information.

\subsubsection{Contextual priming from scripts}

Contextual priming is what we find if a certain situational context is evoked by a story scenario, and different aspects of this context are evident in the narrative. This is related to both the linguistic theory of scripts, and the linguistic and sociopsychological theories of framing and frame semantics (Fillmore, 1976; Goffman, 1979). Using common sense scenarios as priming sources means that we do not evaluate the effects of specific cues (like images). What we did instead was to measure how easily such a prototypical context is evoked, and how much of the narrative content is derived directly from this context.

Our hypotheses are the same as they are for visual priming. Narratives that show a high proportion of script-based context indicate the ability to evoke the respective script, but also point to potential deficits in thinking of narrative elements outside the primed scenario. Alternatively, should the narratives of one of the clinical groups show no use of script-based context, this would suggest dif- ficulties in the application of shared common-sense knowledge, a feature associated with ASD.

\subsection{Experimental Priming Data}

We needed additional data to examine the two different types of priming: For image-based priming, we collected descriptions of the images shown together with the story prompt. In order to compare this with script-based priming, we also collected entities prototypically associated with the given scenarios. Previous research has argued that script data is hard to obtain from standard texts or word embeddings (Regneri et al., 2010), so we decided to use crowdsourcing for both types of data.

Using Amazon Mechanical Turk (AMT), we set up two separate experiments. We collected Image descriptions by presenting the drawings and asking participants for one-word descriptions of the images' contents, encouraging them to name visible entities as well as associated events or moods. For each image, we asked 10 distinct turkers to provide at least 2 and at most 10 words. The participants did not see any verbal description of the picture.

In a similar fashion, we also crowdsourced prototypical script data: For each scenario (both fictional and event-based), we asked which things, people or actions the turkers would typically expect when the given event occurs. We explicitly noted that some scenarios might be fictional and advised the participants to name things they would expect in a story featuring the given scenario. The images were not shown to the participants.

After manually filtering out obvious fraughts, incomprehensible inputs and inputs neglecting the task (15\% overall), we aggregated the data and kept all descriptions that occurred at least twice. We have provided the collected data and the image stimuli in the appendix and as supplementary data.

Table 3 contains some basic figures on the preprocessed data, including the number of words retained for both tasks and the overlap of both lemma sets (as Jaccard score). The overlap between imagebased data and script data indicates prototypicalness: scenarios represented by a more concise inventory of entities show a higher overlap between descriptions derived from pictures and from commonsense scenarios.

\subsection{Measures}

After obtaining textual representations of the priming cues, we related these to our narratives. Technically, we measured the overlap of the AMT data 


\begin{tabular}{lrrr}
\hline Scenario & Image & Script & Jaccard \\
\hline FOREST & 6 & 4 & 0.25 \\
MOUNTAIN & 12 & 7 & 0.19 \\
\hline FREE TIME & 21 & 10 & 0.15 \\
BE SCARED & 16 & 12 & 0.00 \\
BIRTHDAY & 9 & 11 & 0.11 \\
HOLIDAY & 18 & 8 & 0.04 \\
HALLOWEEN & 11 & 7 & 0.06 \\
BE ANGRY & 10 & 7 & 0.06 \\
\hline
\end{tabular}

Table 3: Statistics on crowdsourced priming data, showing the number of words for the two experiments and their overlap measured as Jaccard index.

with the story vocabulary. Considered in isolation, a low overlap is not indicative, because various reasons could explain the absence of crowdsourced vocabulary in the stories (differences in task, age, nationalities, and so on). We did assume, however, that comparing the presence of such vocabulary can positively indicate whether or not the participants do incorporate extra-linguistic context into their stories, and how the groups differ in doing so.

We measured two types of overlap: first we counted how many stories contained any of the primed expressions at all (stories primed):

$$
\text { stories }_{p r}=\frac{\mid\{\text { stories w. AMT vocabulary }\} \mid}{\mid\{\text { all stories }\} \mid}
$$

While this indicated the general prevalence of priming in the participants, we also evaluated the usage of priming-based vocabulary, measuring how many content words of a story $s$ consisted of primingbased data (vocab primed) as follows:

$\operatorname{vocab}_{p r}(s)=\frac{\mid\{\text { content words in } \mathrm{s} \cap \text { AMT data }\} \mid}{\mid\{\text { all content words in } \mathrm{s}\} \mid}$

We defined content words to be all nouns, verbs, adjectives and adverbs, according to POS tagging with CoreNLP (Manning et al., 2014). The extreme cases are that either no content word has a priming source $\left(\operatorname{vocab}_{p r}(s)=0\right)$, or that there is no word without a priming source $\left(\operatorname{vocab}_{p r}(s)=1\right.$, e.g. two words describing the picture). For our evaluation, we calculate $\oslash$ vocab $_{p r}$ as the average primed vocabulary over all stories $s$.

Differences in priming effects between the groups were examined using a $\chi^{2}$-test for stories ${ }_{p r}$ and an independent samples $t$ test for vocab ${ }_{p r}$. As the analysis of the scores necessitated the use of multiple tests, in order to control for a type 1 error, we adopted a more stringent $\alpha$-level, $p<0.01$.

\begin{tabular}{lcccc}
\hline Stimulus type & ASD & DLD & LM & AM \\
\hline Images & 0.54 & 0.58 & 0.70 & 0.73 \\
Scripts & 0.59 & 0.63 & 0.69 & 0.67 \\
\hline
\end{tabular}

Table 4: Overall proportion of stories primed.

\begin{tabular}{llcc}
\hline Group & type & stories $_{p r}$ & $\oslash$ vocab $_{p r}(\mathrm{SD})$ \\
\hline \multirow{2}{*}{ ASD } & GEN & 0.61 & $0.09(0.12)$ \\
& SEN & $\mathbf{0 . 3 9}$ & $0.05(0.10)$ \\
& FIC & $\mathbf{0 . 7 6}$ & $0.07(0.06)$ \\
\hline \multirow{2}{*}{$\mathrm{DLD}$} & GEN & $\mathbf{0 . 5 6}$ & $0.09(0.15)$ \\
& SEN & 0.49 & $\mathbf{0 . 0 7}(0.12)$ \\
& FIC & 0.90 & $0.08(0.06)$ \\
\hline \multirow{2}{*}{ LM } & GEN & 0.72 & $0.09(0.10)$ \\
& SEN & 0.60 & $0.05(0.07)$ \\
& FIC & 0.92 & $0.07(0.05)$ \\
\hline \multirow{2}{*}{ AM } & GEN & 0.77 & $0.08(0.09)$ \\
& SEN & 0.63 & $0.05(0.08)$ \\
& FIC & 0.91 & $0.06(0.03)$ \\
\hline
\end{tabular}

Table 5: The percentage of stories with image-based content and the average proportion of priming-based vocabulary per story, divided by story type.

\subsection{Results}

Table 4 displays the overall results for the scriptbased and the image-based experiment, indicating the proportion of stories primed. Overall, the ASD group exhibited the lowest priming effects. In the image-based experiment, both the ASD and the DLD groups differ significantly from the two control groups, but not from each other. For the script-based condition, the ASD group narrates significantly fewer primed stories than the languagematched group and notably fewer than the agematched group $(p<0.02)$, while the other three groups show no significant difference in this scenario. While it seems that the ASD and the DLD groups only differ for script-based priming, a more detailed examination of the data allows for a more informative differentiation.

\subsection{Image-based Priming}

Table 5 distinguishes the story types and additionally shows the proportion of primed vocabulary. In their fictional stories, the ASD group displayed a significantly lower proportion of primed stories than the other groups, while the proportion of priming based vocabulary does not differ from the control groups. The average use of priming based vocabulary $\left(\oslash \operatorname{vocab}_{p r}\right)$ was higher in the DLD group $(p<0.05)$, but there were no significant differences between any of the other groups. Note that 
the proportion of vocabulary also includes stories without any primed content, so this figure implicitly reflects the proportion of primed stories.

As expected, we find less primed vocabulary in the event narratives: Firstly, the fictional story pictures contained some elements mentioned by nearly all participants (like the alien), showing that the adolescents took this picture as a starting point rather than an optional support. Secondly, the specific event narratives contained stories from the participants' personal histories, for which we expected a smaller priming effect. Most groups indeed distinguished clearly between general and specific narratives. While the stories as a whole contained prototypical concepts in both cases, the proportion of vocabulary originating from priming sources showed an interesting trend: the general narratives contained a similar amount of vocabulary from priming sources in all groups and the specific narratives for the DLD group contained a larger amount than those of all other groups (the difference between GEN and SEN is not significant, while it is significant for all other groups).

These more detailed figures present a different perspective on the number of stories primed: While the average number primed was very similar in both the ASD and the DLD groups and much lower than in the control groups, the ASD group showed a clear distinction between general and specific narratives, and a lower priming sensitivity on average. The DLD group had the same priming sensitivity for the fictional stories we find for the AM and the LM groups, but showed comparable priming effects for specific and general contexts.

As far as the proportion of primed vocabulary is concerned, additional invention of vocabulary (concepts not shown in the picture) for the specific narratives is triggered for the ASD, AM and LM groups but not the DLD group. While on average, the ASD group used priming-based vocabulary in fewer cases than all other groups, the vocabulary was used in an unmarked way if used at all. The DLD group, however, while showing a typical frequency of priming effects, built their specific stories more on the primed concepts than the other groups. For the general event narratives, the DLD group shows less priming sensitivity than any other group, underlining the unusual similarity of the two event variants (GEN and SEN).

\begin{tabular}{llcc}
\hline Group & type & \% stories & $\oslash$ vocab (SD) \\
\hline \multirow{4}{*}{ ASD } & GEN & 0.70 & $0.11(0.12)$ \\
& SEN & $\mathbf{0 . 4 5}$ & $0.05(0.10)$ \\
& FIC & $\mathbf{0 . 6 6}$ & $0.05(0.06)$ \\
\hline \multirow{3}{*}{ DLD } & GEN & $\mathbf{0 . 6 6}$ & $0.11(0.14)$ \\
& SEN & 0.58 & $\mathbf{0 . 0 8}(0.10)$ \\
& FIC & 0.73 & $0.05(0.05)$ \\
\hline \multirow{3}{*}{ LM } & GEN & 0.73 & $0.10(0.09)$ \\
& SEN & 0.63 & $0.06(0.07)$ \\
& FIC & 0.76 & $0.04(0.04)$ \\
\hline \multirow{3}{*}{ AM } & GEN & 0.73 & $0.09(0.08)$ \\
& SEN & 0.63 & $0.05(0.07)$ \\
& FIC & 0.76 & $0.04(0.03)$ \\
\hline
\end{tabular}

Table 6: The percentage of stories with script-based content and the average proportion of priming-based vocabulary per story, divided by story type.

\subsection{Script-based Priming}

Table 6 summarises the results for script-based priming. None of the groups differed significantly in the fictional stories. This was to some degree expected: while there are some prototypical associations with "alien scenarios" of "snowy secluded mountain scenarios", these fictional contexts do not give rise to actual scripts in the sense of prototypical everyday actions. In the event narratives, the average proportion of stories containing contextually primed content was significantly lower in the ASD group than in both of the comparison groups (LM $p<0.01$, AM $p<0.05$ ), while the DLD group does not differ significantly from any group.

Similar to the image-based experiment, both the use of script-based vocabulary and the distinction between the general and specific narratives seems to be related to language competency rather than to ASD (cf. Table 6): While all other groups showed significant differences between general and specific narratives ( $p<0.01$ ), the DLD group showed the least $(p=0.02)$. Comparing the proportions of vocabulary used, the DLD group showed a higher proportion of priming-based vocabulary than the two comparison groups both on average and in the specific event setting $(p<0.01)$.

Both the percentage of narratives with primed content and the proportion of vocabulary from priming cues was, in general, higher for scriptbased priming, even in the fictional narratives. For event narratives, this is due to the nature of general narratives, which contain a prototypical scenario description and thus reflect exactly what we used as priming cues. Relating them to the crowdsourced data analyzes, therefore, not actual priming but 
rather an alignment with concepts from the same scenario. Script-based priming is reflected better in the specific narratives, which contain personal narrations of individual events. Here the average priming level resembles the image-based priming results.

\section{Results and Discussion}

In this section, we interpret our results and review to what extent they answer open questions about priming in adolescents with ASD and with DLD.

\subsection{Priming effects in ASD and DLD}

We compared script-based and visual priming, and the results show notable differences between the groups. On average, the ASD group uses priming cues less frequently than all other groups. This is particularly evident for script-based priming, where the ASD group shows fewer average priming effects than both of the comparison groups, in contrast to the DLD group whose intermediate priming frequency does not differ significantly from any other group (cf. Table 4).

These results indicate either a lack of, or an inability to apply, script-based abstract knowledge, as a typical trait of the ASD group. As it is not evident in either the language-matched group or the group with manifested language development disorder, the script-related phenomena are independent of language ability. Our data suggests that the ASD group can probably access the scripts per se - this is supported by their unmarked priming sensitivity for general narratives. However, they relate their personal stories to the underlying script less frequently than the other groups, which suggests an inability to connect specific content to a broader context. At the same time, the partially low priming sensitivity of the DLD group might simply be a correlate of smaller or atypical vocabulary.

With regard to the extent of which cue-based concepts are used, participants with ASD do not differ from the neurotypical comparison groups: primed content is present in the same proportion of vocabulary. Further, the ASD group and the two comparison groups differentiate between general and specific event narratives in the same way, with more priming cues reflected in the former and fewer in the latter. However, unlike the ASD group, individuals with DLD show a lack of differentiation between general scenario descriptions and specific narratives. Compared to the ASD group, they use fewer priming cues in the general narratives, but they do so more often in the specific case. This means that the overall effect appears similar to the ASD group, but suggests that there may be different underlying causes. Their narratives also contain more cued content than the comparison groups.

Our findings concerning image-based priming complement previous research on language understanding: Yaneva et al. (2015) showed that adults with autism profit more than neurotypical controls from supporting images for text comprehension. In contrast, our study, found that supporting images contributed more rarely to the narrative stories of the ASD group than in all of the neurotypical groups. However, there are important differences between the studies in terms of the age of the participants and the matching of control groups. Not withstanding, the distinction between priming for language understanding and language production remains an interesting area to explore further.

\subsection{Implications and Open Questions}

Our results further the understanding of the use of context in narrative construction. The ASD group makes less use of context in their narratives than all other groups. However, when they do use context, they do so in a typical manner. This is in contrast to the DLD group. While this group also uses context less frequently than the comparison groups, context use is over-proportional when they do so, especially in unexpected cases as in the narratives with personal experiences. These results support previous findings showing that children with ASD demonstrate impairment in priming, but no absolute inability to use contextual information (Henderson et al., 2011). Moreover, they lend support to the theory of weak central coherence which proposes that individuals with ASD have difficulties in processing incoming information in context for global meaning. In contrast, the DLD group shows slightly lower priming sensitivity than the comparison groups, but when they do so, their use of primed content is over-proportional, especially in the specific event narratives.

The indications are that this impairment in priming is independent of language ability and specific to ASD. The DLD group shows a different pattern of impairment, indicating a different underlying aetiology than in the ASD group. The over-proportional use of context by the DLD group might be a compensation for their difficulties in 
language production.

This study delivers only some initial indicators of priming in individuals with ASD and DLD and it has a number of limitations. Firstly, this is not a classical priming study because we are examining language production rather than comprehension, and we have analysed content at the discourse-level rather than word selection or sentential structures. Given this complex task, we employed a simple pragmatic approach for initial analysis. We are aware that our results cannot give a precise account of the extent or nature of priming effects on a discourse level, but this was not the aim of the study. Rather, we targeted a comparative view of different groups, showing how explicit (visual) and implicit (script-based) cues influence the narration style of differently developing adolescents.

The evidence we have found suggests a division of these priming effects which can be either attributed to autism spectrum disorder, or developmental language disorder, or neither of these communication disorders. While this is not theorysound proof of either attribution so far, we have developed some data-informed hypotheses as a source for new questions and further experiments.

\section{Conclusion}

We conclude this paper with a short summary and some suggestions for future work.

\subsection{Summary}

Our study evaluated visual and script-based priming effects in individuals with ASD and DLD, in comparison to neurotypical control groups. We based our analyses on a dataset containing fictional and event-based narratives of adolescents with ASD, DLD and two age-matched and languagematched comparison groups. Additionally, we crowdsourced textual representations of images and scenario descriptions as priming cues. In our experiment, we demonstrated the overlap of these representations with the narratives in our dataset and differentiated the text types for the different groups. Our findings show that, although in general less sensitive to priming cues, adolescents with ASD use these cues either not at all or in the same way as neurotypical peers. In contrast, adolescents with DLD show a typical sensitivity to priming cues in most cases, but exhibit an overproportional use of primed content.

\subsection{Future Work}

To the best of our knowledge, there is no preceding work showing priming effects on language production in adolescents with ASD or DLD. While we have found interesting initial results, there are still many unanswered questions. Further research is needed to test if these findings are replicable. Future work could examine other aspects of priming, in particular differences in priming in all groups between scripts and images.

Future experiments could observe participants' attention while relating the narratives, which would give real-time indications of a priming mechanism. More data containing both different languages and offering other sources of extra-linguistic priming would help to explore the circumstances that facilitate or inhibit priming effects in individuals with ASD. With regards to priming effects in adolescents with DLD, analysing their capability to distinguish between general and specific event narratives could also be fruitful area of research.

\section{References}

APA. 2013. Diagnostic and statistical manual of mental disorders: DSM-5, 5th ed. edition. American Psychiatric Association, Washington, DC.

Dorothy V.M. Bishop, Margaret J. Snowling, Paul A. Thompson, Trisha Greenhalgh, , and the CATALISE-2 consortium. 2017. Phase 2 of catalise: a multinational and multidisciplinary delphi consensus study of problems with language development: Terminology. Journal of Child Psychology and Psychiatry, 58(10):1068-1080.

D.V.M. Bishop, Margaret Snowling, Paul Thompson, Trisha Greenhalgh, C. Adams, Lisa Archibald, G. Baird, A. Bauer, J. Bellair, Christopher Boyle, Elizabeth Brownlie, Glenn Carter, B. Clark, Judy Clegg, Nancy Cohen, Gualtiero Nunzi Conti, Julie Dockrell, J. Dunn, Susan Ebbels, and Andrew Whitehouse. 2016. Catalise: A multinational and multidisciplinary delphi consensus study. identifying language impairments in children. PLOS ONE, 11.

Joan Bresnan, Anna Cueni, Tatiana Nikitina, and Harald Baayen. 2007. Predicting the dative alternation. Royal Netherlands Academy of Science.

Gina Conti-Ramsden and Nicola Botting. 1999. Classification of children with specific language impairment. Journal of Speech, Language, and Hearing Research, 42(5):1195-1204.

Walter Daelemans. 2013. Explanation in computational stylometry. In Proc. of CICLing'13. 
Luna De Bruyne, Ben Verhoeven, and Walter Daelemans. 2018. Stylometric text analysis for dutchspeaking adolescents with autism spectrum disorder. Computational Linguistics in the Netherlands, 8:323.

Gary S. Dell and Franklin Chang. 2014. The pchain: Relating sentence production and its disorders to comprehension and acquisition. Philosophical Transactions of the Royal Society B: Biological Sciences, 369(1634).

Julie Dockrell, Geoff Lindsay, Olympia Palikara, and Mairi Cullen. 2007. Raising the achievements of children and young people with specific speech and language difficulties and other special educational needs through school to work and college.

Lloyd M. Dunn and Leota M. Dunn. 1997. The British Picture Vocabulary Scale Second Edition (BPVS II). Windsor Berkshire: NFER-NELSON Publication Company.

Colin Elliot, Pauline Smith, and Kay McCullouch. 1996. The British Ability Scales II (BASII). Windsor Berkshire: NFER-NELSON Publication Company.

Charles J. Fillmore. 1976. Frame semantics and the nature of language. Annals of the New York Academy of Sciences, 280(1):20-32.

Anouschka Foltz, Kristina Thiele, Dunja Kashnitz, and Prisca Stenneken. 2015. Children's syntacticpriming magnitude: lexical factors and participant characteristics. Journal of Child Language, 42(4):932-945.

Courtenay Frazier Norbury, Tracey Gemmell, and Rhea Paul. 2013. Pragmatics abilities in narrative production: A cross-disorder comparison. Journal of child language, 41:1-26.

Uta. Frith. 1989. Autism : explaining the enigma. Basil Blackwell, Oxford, UK; Cambridge, MA, USA.

Keyur Gabani, Melissa Sherman, Thamar Solorio, Yang Liu, Lisa Bedore, and Elizabeth Peña. 2009. A corpus-based approach for the prediction of language impairment in monolingual english and spanish-english bilingual children. In Proc. of NAACL-HLT 2009.

Keyur Gabani, Thamar Solorio, Yang Liu, Khairunnisa Hassanali, and Christine A. Dollaghan. 2011. Exploring a corpus-based approach for detecting language impairment in monolingual english-speaking children. Artif. Intell. Med., 53(3).

Michael S. Gaffrey, Natalia M. Kleinhans, Frank Haist, Natacha Akshoomoff, Ashley Campbell, Eric Courchesne, and Ralph-Axel Müller. 2007. A typical participation of visual cortex during word processing in autism: An fmri study of semantic decision. Neuropsychologia, 45(8):1672 - 1684.
Maria Garraffa, Moreno I. Coco, and Holly P. Branigan. 2015. Effects of immediate and cumulative syntactic experience in language impairment: Evidence from priming of subject relatives in children with sli. Language Learning and Development, 11(1):18-40.

Maria Garraffa, Moreno I Coco, and Holly P Branigan. 2018. Impaired implicit learning of syntactic structure in children with developmental language disorder: Evidence from syntactic priming. Autism \& Developmental Language Impairments, 3:2396941518779939.

Erving Goffman. 1979. Frame analysis: An essay on the organization of experience. Philosophy and Phenomenological Research, 39(4):601-602.

Micah Goldwater, Marc Tomlinson, Catharine Echols, and Bradley Love. 2011. Structural priming as structure-mapping: Children use analogies from previous utterances to guide sentence production. Cognitive science, 35:156-70.

Shelley Gray, Elena Plante, Rebecca Vance, and Mary Henrichsen. 1999. The diagnostic accuracy of four vocabulary tests administered to preschool-age children. Language, Speech, and Hearing Services in Schools, 30(2):196-206.

Suzanne Hala, Penny Pexman, and Melanie Glenwright. 2007. Priming the meaning of homographs in typically developing children and children with autism. Journal of autism and developmental disorders, 37:329-40.

Francesca Happe and Uta Frith. 2006. The weak coherence account: Detail-focused cognitive style in autism spectrum disorders. Journal of autism and developmental disorders, 36:5-25.

L M Henderson, P J Clarke, and M J Snowling. 2011. Accessing and selecting word meaning in autism spectrum disorder. Journal of Child Psychology and Psychiatry, 52(9):964-973.

Yoko Kamio, Diana Robins, Elizabeth Kelley, Brook Swainson, and Deborah Fein. 2007. Atypical lexi$\mathrm{cal} /$ semantic processing in high-functioning autism spectrum disorders without early language delay. Journal of Autism and Developmental Disorders, 37(6):1116-1122.

EVAN KIDD. 2012. Individual differences in syntactic priming in language acquisition. Applied Psycholinguistics, 33(2):393-418.

Diane King, Julie Dockrell, and Morag Stuart. 2014. Constructing fictional stories: a study of story narratives by children with autistic spectrum disorder. Research in developmental disabilities, 35(10).

Diane King, Julie E Dockrell, and Morag Stuart. 2013. Event narratives in 11-14 year olds with autistic spectrum disorder. International Journal of Language \& Communication Disorders, 48(5). 
Diane King and Olympia Palikara. 2018a. Assessing language skills in adolescents with autism spectrum disorder. Child Language Teaching and Therapy, 34(2):101-113.

Diane King and Olympia Palikara. 2018b. Dataset with narratives by children with developmental language disorder. In Preparation.

Maryellen MacDonald. 2013. How language production shapes language form and comprehension. Frontiers in Psychology, 4:226.

Christopher D. Manning, Mihai Surdeanu, John Bauer, Jenny Finkel, Steven J. Bethard, and David McClosky. 2014. The Stanford CoreNLP natural language processing toolkit. In Proc. of ACL 2014: System Demonstrations.

Maria Manolitsi and Nicola Botting. 2011. Language abilities in children with autism and language impairment: using narrative as a additional source of clinical information. Child Language Teaching and Therapy, 27(1):39-55.

Carol A. Miller and Patricia Deevy. 2006. Structural priming in children with and without specific language impairment. Clinical Linguistics \& Phonetics, 20(5):387-399. PMID: 16728335.

Laurent Mottron, Karine Morasse, and Sylvie Belleville. 2001. A study of memory functioning in individuals with autism. Journal of child psychology and psychiatry, and allied disciplines, 42:253-60.

Courtenay Frazier Norbury and Dorothy V. M. Bishop. 2003. Narrative skills of children with communication impairments. International Journal of Language \& Communication Disorders, 38(3):287-313.

Emily T Prud'hommeaux, Brian Roark, Lois M Black, and Jan van Santen. 2011. Classification of atypical language in autism. ACL HLT 2011.

Pia Rämä, Louah Sirri, and Josette Serres. 2013. Development of lexical-semantic language system: N400 priming effect for spoken words in 18- and 24-month old children. Brain and Language, 125(1):1-10.

Michaela Regneri and Diane King. 2015. Automatically evaluating atypical language in narratives by children with autistic spectrum disorder. In Proc. of NLPCS 2014.

Michaela Regneri and Diane King. 2016. Automated discourse analysis of narrations by adolescents with autistic spectrum disorder. In Proceedings of the 7th Workshop on Cognitive Aspects of Computational Language Learning, pages 1-9. Association for Computational Linguistics.

Michaela Regneri, Alexander Koller, and Manfred Pinkal. 2010. Learning script knowledge with web experiments. In Proceedings of ACL 2010, Uppsala, Sweden. Association for Computational Linguistics.
Stefano Rezzonico, Xi Chen, Patricia L. Cleave, Janice Greenberg, Kathleen Hipfner-Boucher, Carla J. Johnson, Trelani Milburn, Janette Pelletier, Elaine Weitzman, and Luigi Girolametto. 2015. Oral narratives in monolingual and bilingual preschoolers with sli. International Journal of Language \& Communication Disorders, 50(6):830-841.

Mabel L. Rice and Lesa Hoffman. 2015. Predicting vocabulary growth in children with and without specific language impairment: A longitudinal study from 2;6 to 21 years of age. Journal of Speech Language and Hearing Research, 58(2):345-359.

Mabel L Rice, Filip Smolik, Denise Perpich, Travis Thompson, Nathan Rytting, and Megan Blossom. 2010. Mean length of utterance levels in 6-month intervals for children 3 to 9 years with and without language impairments. Journal of Speech, Language, and Hearing Research.

Masoud Rouhizadeh, Emily Prud'hommeaux, Brian Roark, and Jan van Santen. 2013. Distributional semantic models for the evaluation of disordered language. In Proc. of NAACL-HLT 2013.

Masoud Rouhizadeh, Emily Prud'Hommeaux, Jan Van Santen, and Richard Sproat. 2015. Measuring idiosyncratic interests in children with autism. In Proc. of ACL 2015.

Roger C. Schank and Robert P. Abelson. 1977. Scripts, Plans, Goals and Understanding. Lawrence Erlbaum, Hillsdale, NJ.

Eleanor Semel, Elisabeth .H Wiig, and Wayne Secord. 2006. Clinical Evaluation of Language Fundamentals (CELF-4 UK), fourth edition uk edition. Pearson Assessment.

Nancy L Stein and Elizabeth R Albro. 1997. Building complexity and coherence: Children's use of of goalstructured knowledge in telling stories. Narrative development: Six approaches, page 5.

Bruce Tomblin. 2011. Co-morbidity of autism and sli: kinds, kin and complexity. International Journal of Language \& Communication Disorders, 46(2):127137.

D. Wetherell, N. Botting, and G. Conti-Ramsden. 2007. Narrative in adolescent specific language impairment (sli): a comparison with peers across two different narrative genres. International Journal of Language \& Communication Disorders, 42(5):583 -605 .

Victoria Yaneva, Irina Temnikova, and Ruslan Mitkov. 2015. Accessible texts for autism: An eye-tracking study. In Proceedings of the 17th International ACM SIGACCESS Conference on Computers \& Accessibility, ASSETS '15, pages 49-57, New York, NY, USA. Association for Computing Machinery. 


\section{A Appendix: Story Corpus Excerpt}

We are providing the complete story corpus as supplementary material, together with the images and the crowd-sourced language data. In this section, to give an impression of the data, we give examples from this corpus alongside the pictures and prompts presented as stimuli. These include some of the transcribed fictional narratives (King et al., 2014) and some exemplary event narratives (King et al., 2013) from each of the participating groups. The transcriptions preserve all (partially ungrammatical) artifacts of the original spontaneous speech. In some cases, the stories were interrupted by intermediary examiner prompts, which we omit for brevity. Slashes ("P") indicate utterance boundaries. We present the instructions used to collect the original data, and include the images we evaluated as priming sources, which were shown to the subjects in the respective experimental setup. From the story corpus, we present two examples from the ASD and two from the DLD group for each story stem, and one example per control group. Additionally, we show the crowdsourced textual priming cues (for a complete version, see the supplementary data of this paper).

\section{A.1 Corpus Examples: Fictional Narratives}

\section{A.1.1 Instructions for participants}

The following shows the instructions given to the participants by King et al. (2014) to collect the story corpus:

I am going to start a story. I'd like you to continue and finish the story. You can use the picture to help you if you like. First, we're going to try a practice one:

The boy was swimming in the river. On a rock, he saw a strange creature, half man, half fish.

For the corpus collection, the participants were then presented with the story stems shown in the following.

\section{A.1.2 The Forest story}

The boy ran into the forest. He looked ahead of him and saw a little green man in a spaceship.

\section{ASD}

Example 1: the spaceship was quite small. / And the alien was about the size of a small cat. / And it was friendly. / but it didn't really understand how

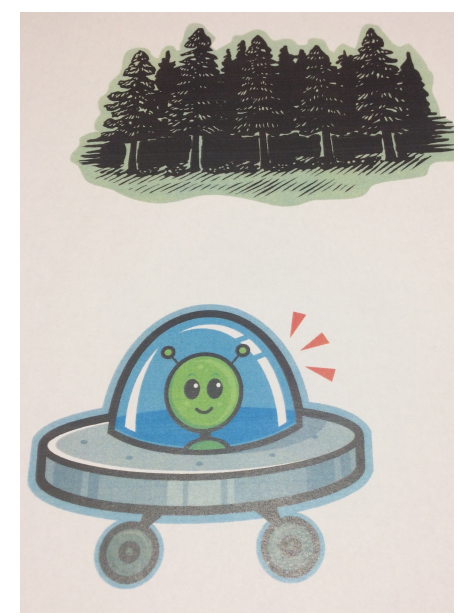

Figure 1: Picture for the Forest story

humans said hello. / So it thought, to say 'hello' you had to vaporise the person in front of you. I and then the boy ran away, shut his door and then decided not to drink anymore whisky or beer.

ASD Example 2: The green man had three eyes. / It had claws and fangs. / It looked at him and ran into the spaceship. / Out came three more green men carrying laser guns, dun dun dun.

\section{DLD}

Example 1: The boy. / he just died from a alien . / Okay maybe he banged his head. / and the alien talk XX. / maybe he was unconscious . / I think the alien was experimenting him . / He was out of space for years and years and years . / and he came back like this. / The end. / Just like that. / He turned to them. / he turned to aliens . / To a evil one . / He have a big big brain. / Having one of those things and pale really long, and some legs . I tall, sharp teeth like, and red eyes, as very scary as anything . / and then people saw that. / and they made it as a story . / that they say that in the forest you'll see they named that animal named by the anaconda. / I mean the an part an part the an perd . / The an pert. / Yeah but they say that that is a story. / but it was real . / but I think aliens they were . / he was trying to call them. / but he could't . / he did this for years. / He was standing in the forest for years and years and years and years and years . / but he's never gaingame, never been older, ever . / Yeah. / And if anybody goes there and see them, they can't go back. / Yeah, if they go in they can't go out. / So that's the end. / The end.

DLD Example 2: Okay. $\mathrm{He}$ runs over to 
the spaceship. / And the green man looks behind him and the person gets freaked out. / Trys to run away. / But then the green man captures him in his spaceship and flies off. / And then they do tests on him. / And with stuff. / And then they put him back into the forest. / And then he runs somewhere else. / And then, his mum sees him. / And quickly grabs him and brings him home. / Ya.

\section{Language-Matched Controls:}

He was shocked at first because he didn't know what it is. / So he walked up. / and he got suck/ed in by a tractor beam. / and he found himself in a UFO. / he was surround/ed by weird looking creatures like aliens. / and they started speaking like this unknown language to him. / and he couldn't understand a thing about them. / So he tried to escape. / he ran away. / but he couldn't cos-because wherever he went he was surround/ed by aliens. / and they eventually capture/ed him and took him off to Mars. /

\section{Age-Matched Controls:}

The little green man waved at him and yelled at him to come and help him with his spaceship which had crashed into the forest after he had lost all his fuel. / The little boy went up to him and said that he didn't know what much he could do because he's obviously not very talented at fixing alien space craft. / so he ran out of the forest went to go get the nearest person he could see. / He then brought him back to the spaceship where the little green man was waiting. / and the man he'd brought back was absolutely amazed. / he went up to the little alien and started speaking with him. / And then after a while he persuaded it to come out of it's spaceship. / and it went to meet the locals who were very very amazed. / and then all the people joined together. / and they pulled his spaceship out of the mud. / and he flew back in it to mars.

\section{A.1.3 The Mountain story}

When the girl climbed up the mountain, she saw, hidden among the trees, a little wooden house covered in snow.

\section{ASD}

Example 1: She went up to the mountain to see the house. / She went inside and had a cup of tea / After that she can't get out because the snow block/ed the door. / And the men came came in and broke it. / but snow came again. / and then she was stuck. / That's it.

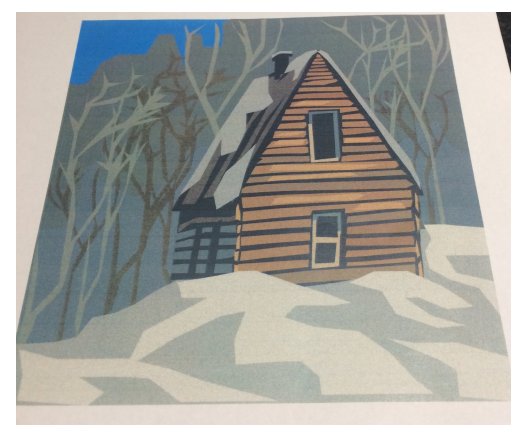

Figure 2: Picture for the Mountain story

ASD Example 2: the snow house was was a zombie. / and the zombie / he went up to the door. / and the zombie scared him. / and the zombie went to chase the girl. / and the girl ran away to to her grandma that was climbing up the mountain. / and she screamed and jumped off the mountain

\section{DLD}

Example 1: The house looked really beautiful to live in. / They were looking everywhere. / They saw no one in. / so they thought they could live in it . / That's it. / (That's) I said that's it. / Nothing else. / I just want to hurry.

DLD Example 2: Yep. / And she thought I'm gonna go and check it out . / And went up to the house. / And walk inside. / Then she saw no lights. / It was really really dark. / The end. / And then she couldn't find the light. / and she was getting really scared. / And, yeah, the end.

\section{Language-Matched Controls:}

She saw it was abandoned. / so she went down to see what it was like. / She peeked inside the window. / and inside there was a pixie . / and then the pixie saw the girl and said 'go away from my window'. / and then he threw a bowl of soup over her. / the little girl went home and said: 'daddy there was a pixie who threw some soup over me'. / and then the dad said 'don't be silly'. / stop telling your little stories'.

\section{Age-Matched Controls:}

She walked towards the house. / the house lit up. / lights switched on. / She knocked on the door. / she was cold. / she asked if she could come in. / There was a strange lady come to the door, pimples and spots all over her, mouldy ugly hair and very very small. / she went in. / the lady was actually a witch in disguise. / She grabbed the girl and threw her 
into the oven. / her friend had also came into the house five minutes later and seen her in the oven. / She had pushed the witch over, got her out and ran off. / they reported it all to the police. / The police came up the next day. / The house was not there.

\section{A.2 Corpus Examples: Event Narratives}

For each scenario, we present a general and a specific example from both the ASD and the DLD group, and selected examples (either general or specific) from the control groups.

\section{A.2.1 Instructions for participants}

The following shows the instructions given to the participants by King et al. (2013) to collect the event narrative corpus. Both the general and the specific condition were first practiced with one scenario:

I'm going to ask you to talk about things that people usually do. I'm also going to show you some pictures which you can use to help you if you like. First we'll have a practice question: What usually happens when someone goes shopping?

The prompt for each scenario was a question referring to the scenario. For the general case, the prompt was either about "people" (like in the example) or about "(n) year olds", with $n$ being the age of the child.

The specific narratives were prompted with a question referring to a specific experience of the subject, again with a practice question:

I'm going to ask you to talk a bit about yourself and things that have happened to you. I'm also going to show you some pictures which you can use to help you if you like. First we'll have a practice question: Can you tell me about a time when you went shopping?

All other prompts then were worded as "Can you tell me about one time when you..." followed by the respective scenario.

\section{A.2.2 Spending free time}

(The Story prompt for the specific question in this scenario did not ask for a specific event, but rather for an aggregated answer: "What do you usually to in your free time?")

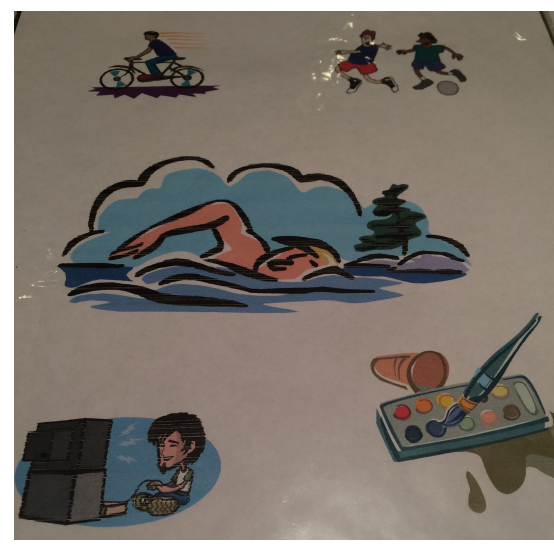

Figure 3: Picture for the free time scenario

\section{ASD}

General Example: They paint any pictures they want, that they like. / (Like say) if you wanna try and make a very good artist (like say) a big picture (of er) with (er like) angels singing or something like that / So you try and make it beautiful

ASD Specific Example: I go biking / I go to football / Sometimes I go swimming in the seaside and (uh) in the pool / After that I watch TV and play on my gameboy / And sometimes I (paint) paint / Yeh

\section{DLD}

General Example: Play games / Watch TV / (Um, they) they go out / (They go foot) they do football DLD Specific Example: In my free time. / I normally spend time with my friends playing games. / Sometimes I go to their house. / And that's all. / Yeah.

\section{Control - language-matched, general:}

(Twelve year old um) Play computer games or play sports or do any other activities that they like doing / maybe drama class / I don't know / Ballet, things like that.

\section{A.2.3 Being scared}

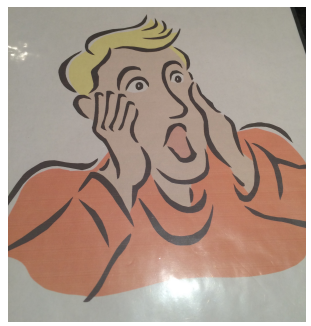

Figure 4: Picture for the scared scenario 
ASD

General Example: They start to sweat / (So I) I know there's the release of a chemical inside the body / And I know that / (um) I can't really think of anything else.

ASD Specific Example: When I was watching this movie called "Alien" when I was about five / (Not) not a very good movie to watch when you're five / Maybe now but not then / It was really scary

DLD

General Example: They're like, haahh oh my god / They are screaming / Running / That, all of that / Yeah, that's also, next one please

DLD Specific Example: Scary life / Zombie / $\mathrm{Hmm} / \mathrm{Hmm} /$

\section{Control - language-matched, specific:}

(Uh) this morning, when I went into the bathroom, there was a big hornet on the ceiling / so I got my mum to kill it / (Uh) not really

\section{A.2.4 Having a birthday}

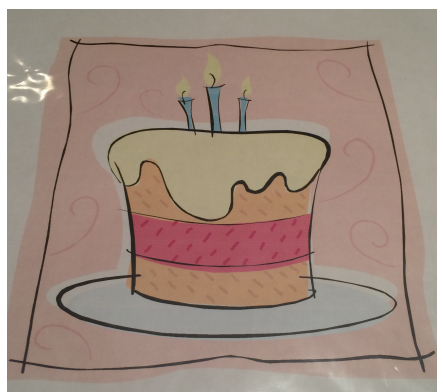

Figure 5: Picture for the birthday scenario

\section{ASD}

General Example: They blow out candles / Well, that's all I did but I don't know what other people would do on their birthday

ASD Specific Example: (My last one) on my last birthday I went up to York national railway museum / I saw trains / I saw trains (in) in the museum / Just for the birthday weekend really /

\section{DLD}

General Example: (Go to like), to go to a holiday / Legoland / I went to Legoland! Go to the funfair / go to see Lego / just build lego / every lego like you do, even here / You went, and even see the other water / Get all your presents / Yeah /

DLD Specific Example: Blowing candle.
Control - age-matched, general:

They have a huge party / bring friends / have cake / (have) get birthday presents from their family / if they have enough money, save up and buy something they really want like a bike, xbox, games, (um) or just spend it on something nice (they really) they've always wanted, I thought / They could sleep out

\section{A.2.5 Going on holdiay}

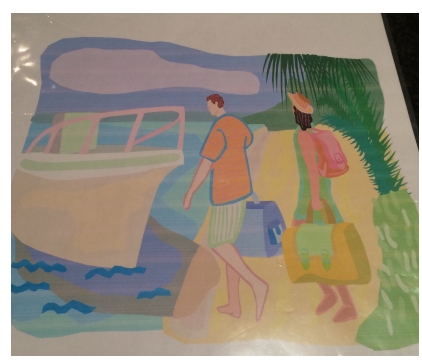

Figure 6: Picture for the holiday scenario

ASD

General Example: A different country, pack stuff, and take clothes.

ASD Specific Example: A different country, pack stuff, and take clothes / yeh yeh yeh / I love holidays / get a day of school / school / I like schools / I (like like to) liked last years / liked last years holiday / no / no / lets stop

\section{DLD}

General Example: Sometimes they get boat / Or they have their drink or something in the boat and have fun there / See dolphins if in that country / some countries have dolphins, some don't

DLD Specific Example: On the boat / Sand, ocean / Beach / suitcase / bag / hat / swimming shorts and bikini

\section{Control - age-matched, specific}

(Uh) when I went on holiday I went to Portugal to my auntie because she was having her wedding anniversary / and we had a big party / and I went for four weeks / (Um) I went swimming in the sea / and (I got) I came out blue

\section{A.2.6 Halloween}

ASD

General Example: Carve pumpkins / Don't know / (uh).

ASD Specific Example: Handed out candy to people who came knocking at my door / That's it 


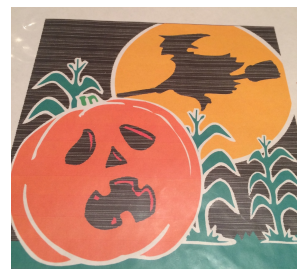

Figure 7: Picture for the halloween scenario

\section{DLD}

General Example: They dress up / And they go trick or treating / And someone knocks on your door you got to give them sweets / dunno

DLD Specific Example: On Halloween I (put up) helped my mum put up pumpkin / And then I went trick or treating

\section{Control - language-matched, general:}

Usually, familys who aren't going out will always buy (like) sweets / and usually to show that they're takeing part they have a pumpkin with a candle inside / And people dress and go to other peoples' houses with bags to collect sweets

\section{A.2.7 Being Angry}

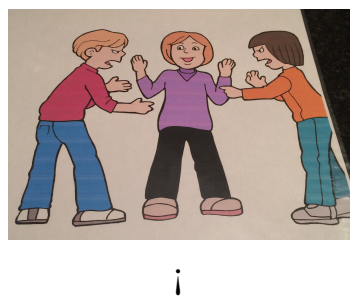

Figure 8: Picture for the angry scenario

\section{ASD}

General Example: They either stop being friends with them or something / or they get into a fight / Start japing at each other

ASD Specific Example: I was angry / Beat up someone / And beat up someone else / Be nasty

\section{DLD}

General Example: Mmm, hating each other. DLD Specific Example: Somebody annoying / Somebody annoying

\section{Control - age-matched, specific:}

(Um) once I got angry with my friend (um) because (um well) he was geting angry with me / so I got angry with him really / (Uh) I think it was because (um) he wasn't being that nice to anyone that day so he got angry with me (so) for some reason / I can't really remember

\section{B Supplementary Data}

We provide the priming stimuli we evaluated in this study (images and data collected on mechanical turk). The data is accessible under the following url: https://imagesandimagination.

s3.eu-central-1.amazonaws.com/

ImagesAndImagination_Supplements.zip

The supplementary material contains a table (priming_amt_data.tsv) as tab-separated values with the following columns:

- Scenario id: An integer (1-8)

- type: event (1-6) or fictional (7 \& 8)

- name: A textual description of the scenario

- script entities: Entities collected via AMT as prototypically occurring in the given scenario, comma-separated (no spaces)

- image entities: Entities collected via AMT that describe the given image

- image file: name of the image file for the given scenario

The textual priming cues collected for the two experiments are additionally shown in table 7 . 


\begin{tabular}{|c|c|c|}
\hline Scenario name & Script-based Cues & Image-based Cues \\
\hline spending free time & $\begin{array}{l}\text { play, video game, friend, football, tele- } \\
\text { vision, swim, run, park, dance, bike }\end{array}$ & $\begin{array}{l}\text { swim, water, paint, brush, tree, player, } \\
\text { game, football, cycle, ball, tv, soccer, } \\
\text { play, paint, men, glass, entertainment, } \\
\text { computer, bike, art, cloud }\end{array}$ \\
\hline being scared & $\begin{array}{l}\text { cry, hide, gun, threat, scary, quietness, } \\
\text { movie, mother, heavy breath, child, } \\
\text { bully, burglar }\end{array}$ & $\begin{array}{l}\text { surprise, man, fear, face, sweater, sur- } \\
\text { prised, shirt, serious, painting, orange, } \\
\text { hair, framework, feeling, color, open, } \\
\text { blonde }\end{array}$ \\
\hline having a birthday & $\begin{array}{l}\text { cake, balloons, party ball, music, gift, } \\
\text { candle, dance, friend, present, game, } \\
\text { family }\end{array}$ & $\begin{array}{l}\text { cake, birthday, plate, candle, celebra- } \\
\text { tion, cream, chart, flame, flavour }\end{array}$ \\
\hline going on holiday & $\begin{array}{l}\text { family, friend, food, drink, beach, suit- } \\
\text { case, music, hotel }\end{array}$ & $\begin{array}{l}\text { boat, woman, tree, ship, man, sea, water, } \\
\text { vacation, suitcase, sand, beach, trip, sky, } \\
\text { luggage, bag, walk, palm, cloud }\end{array}$ \\
\hline halloween & $\begin{array}{l}\text { candy, costume, treat, pumpkin, skele- } \\
\text { ton, party, trick }\end{array}$ & $\begin{array}{l}\text { pumpkin, witch, moon, hat, halloween, } \\
\text { scary, night, jack-o-lantern, horror, fear, } \\
\text { plant }\end{array}$ \\
\hline being angry & $\begin{array}{l}\text { fight, frowning, crying, yelling, upset, } \\
\text { tension, shouting }\end{array}$ & $\begin{array}{l}\text { arguing, boy, smile, pointing, playing, } \\
\text { girl, fight, child, anger, argument }\end{array}$ \\
\hline forest story & $\begin{array}{l}\text { snow, tree, rocks, people, house, fear, } \\
\text { dog }\end{array}$ & $\begin{array}{l}\text { forest, alien, ufo, tree, extra terrestrial, } \\
\text { spaceship }\end{array}$ \\
\hline mountain story & tree, animals, alien, abduction & $\begin{array}{l}\text { snow, wood, tree, cabin, forest, win- } \\
\text { dows, quiet, mountain, hut, house, } \\
\text { home, cold }\end{array}$ \\
\hline
\end{tabular}

Table 7: Priming cues for the different story scenarios crowdsourced via mechanical turk and filtered (cf. Sec. 3) 